\title{
Stress Hormone Exposure Reduces mGluR5 Expression in the Nucleus Accumbens: Functional Implications for Interoceptive Sensitivity to Alcohol
}

\author{
Joyce Besheer*, 1,2,3, Kristen R Fisher', Anel A Jaramillo',2, Suzanne Frisbee' and Reginald Cannady ${ }^{1,2}$ \\ 'Bowles Center for Alcohol Studies, University of North Carolina at Chapel Hill, Chapel Hill, NC, USA; ${ }^{2}$ Curriculum in Neurobiology, University of \\ North Carolina at Chapel Hill, Chapel Hill, NC, USA; ${ }^{3}$ Department of Psychiatry, University of North Carolina at Chapel Hill, Chapel Hill, NC, USA
}

\begin{abstract}
Escalations in alcohol drinking associated with experiencing stressful life events and chronic life stressors may be related to altered sensitivity to the interoceptive/subjective effects of alcohol. Indeed, through the use of drug discrimination methods, rats show decreased sensitivity to the discriminative stimulus (interoceptive) effects of alcohol following exposure to the stress hormone corticosterone (CORT). This exposure produces heightened elevations in plasma CORT levels (eg, as may be experienced by an individual during stressful episodes). We hypothesized that decreased sensitivity to alcohol may be related, in part, to changes in metabotropic glutamate receptors-subtype 5 (mGluR5) in the nucleus accumbens, as these receptors in this brain region are known to regulate the discriminative stimulus effects of alcohol. In the accumbens, we found reduced mGluR5 expression (immunohistochemistry and Western blot) and decreased neural activation (as measured by c-Fos immunohistochemistry) in response to a moderate alcohol dose (I g/kg) following CORT exposure (7 days). The functional role of these CORT-induced adaptations in relation to the discriminative stimulus effects of alcohol was confirmed, as both the systemic administration of 3-Cyano- $\mathrm{N}$-(I,3-diphenyl-IH-pyrazol-5-yl)benzamide (CDPPB) an mGluR5 positive allosteric modulator and the intra-accumbens administration of (R,S)-2-Amino-2-(2-chloro-5-hydroxyphenyl)acetic acid sodium salt (CHPG) an mGluR5 agonist restored sensitivity to alcohol in discrimination-trained rats. These results suggest that activation of mGluR5 may alleviate the functional impact of the CORT-induced downregulation of mGluR5 in relation to sensitivity to alcohol. Understanding the contribution of such neuroadaptations to the interoceptive effects of alcohol may enrich our understanding of potential changes in subjective sensitivity to alcohol during stressful episodes.

Neuropsychopharmacology (20I4) 39, 2376-2386; doi: I 0.I038/npp.20 I4.85; published online 7 May 2014
\end{abstract}

\section{INTRODUCTION}

Exposure to stressors, both physical/emotional (eg, restraint/social stress) and physiological (glucocorticoid exposure), can impact several neurobiological systems including glutamatergic signaling pathways. The presence of dysregulated glutamatergic systems is indicated by adaptations in glutamate levels and/or targets following stress or exogenous corticosterone (CORT) exposure (see Popoli et al, 2012). Much of the existing literature examining stressor-induced alterations of glutamate signaling has focused on adaptations in ionotropic glutamate receptors (AMPA and NMDA). However, the impact of stress on adaptations in metabotropic glutamate receptors (mGluRs) has not been studied as extensively.

\footnotetext{
* Correspondence: Dr J Besheer, Bowles Center for Alcohol Studies, Thurston-Bowles Building; CB\#7178, University of North Carolina at Chapel Hill, Chapel Hill, NC 27599, USA, Tel: + I 919843 9478, Fax: + I 919966 5679, E-mail: jbesheer@med.unc.edu

Received 24 January 2014; revised II March 2014; accepted 2 April 2014; accepted article preview online 9 April 2014
}

mGluRs are G-protein-coupled receptors and are well characterized for their relatively modest modulatory role in glutamate neurotransmission (Niswender and Conn, 2010); a feature that makes them attractive therapeutic targets for treating neuropsychiatric disorders (Krystal et al, 2010). In addition to other mGluR subtypes, preclinical studies support a role for the Group I subtype, mGluR5, in modulating drug- and alcohol-related behaviors (Olive, 2009). Further, mGluR5 activity in the nucleus accumbens has a critical role in drug- and alcohol-seeking behaviors, reinforcement processes, and synaptic plasticity (Besheer et al, 2010; Cozzoli et al, 2012; D'Ascenzo et al, 2009; D'Souza and Markou, 2011; Huang and Hsu, 2012; Moussawi et al, 2009; Schotanus and Chergui, 2008; Sinclair et al, 2012). While exposure to stressors can contribute to alcohol-drinking and -seeking behaviors (Becker et al, 2011), it is not known whether mGluR5 systems are impacted by this exposure and consequently whether the ability of mGluR5 to functionally modulate alcohol-related behaviors is affected. Therefore, examination of stressor-induced adaptations in accumbens mGluR5 systems and behavioral mechanisms that have potential to influence drug-taking and -seeking behaviors is critical. 
One such behavioral mechanism relates to interoceptive/ subjective drug effects. In preclinical models, interoceptive drug cues can serve as potent modulators of drug-taking, seeking, and relapse (Banks et al, 2007; Mihindou et al, 2011; Schenk and Partridge, 1999; Stolerman, 1992; Wise et al, 2008). Drug discrimination procedures are commonly used in both humans and animals as an index of interoceptive (discriminative stimulus) effects of drugs. Using these procedures, preclinical studies have determined that sensitivity to the interoceptive effects of alcohol requires, in part, mGluR5 activity in the nucleus accumbens. This has been determined from studies in which pharmacological antagonism of mGluR5 results in decreased sensitivity to the interoceptive effects of alcohol (Besheer et al, 2009; Besheer and Hodge, 2005; Besheer et al, 2006). Interestingly, exposure (7-day) to the stress hormone corticosterone (CORT) results in decreased sensitivity to the interoceptive effects of alcohol in rats (Besheer et al, 2012b), a similar behavioral pattern to that observed following mGluR5 antagonism. At present, there is rich literature showing stress-induced neuroadaptations in the hippocampus or prefrontal cortex (see for example, Popoli et al, 2012). These regions highly express glucocorticoid and mineralocorticoid receptors (Herman, 1993; McEwen et al, 1986) and have dense glutamatergic projections to the nucleus accumbens (Friedman et al, 2002; Phillipson and Griffiths, 1985). Consequently, the presence of mGluR5related adaptations in the nucleus accumbens is highly probable. Accordingly, we hypothesized that decreased sensitivity to alcohol following CORT exposure may be related to a downregulation of mGluR5 in the nucleus accumbens.

To test this hypothesis, we first sought to determine the effects of stress hormone exposure (7-day CORT) on mGluR5 expression in the nucleus accumbens. Next, in order to determine whether CORT exposure alters neuronal response to a moderate alcohol dose $(1 \mathrm{~g} / \mathrm{kg}, \mathrm{IG})$ in the striatum, c-Fos immunohistochemistry was examined. Finally, drug discrimination procedures were used to establish whether systemic and site-specific intra-accumbens pharmacological activation of mGluR5 could functionally restore sensitivity to alcohol following CORT exposure. Overall, these studies aim to better understand the functional adaptations in accumbal mGluR5 as a consequence of prolonged and heightened elevations in CORT in relation to sensitivity to alcohol interoceptive cues.

\section{MATERIALS AND METHODS}

Please refer to Supplementary Methods for additional procedural details.

\section{Animals}

Individually housed male Long-Evans rats (Harlan Sprague-Dawley, Indianapolis, IN) were used in these studies. All rats were weighed and handled daily for at least 1 week before testing. To match the alcohol discrimination experiments, food intake was restricted to $\sim 16 \mathrm{~g}$ of food/day to maintain body weight (325-340 g) for all experiments. Water was available ad libitum in the home cage unless noted. Experiments were conducted during the light cycle, and the colony room was maintained on a 12-h light/dark cycle. Animals were under continuous care and monitoring by veterinary staff from the Division of Laboratory Animal Medicine at UNC-Chapel Hill. All procedures were conducted in accordance with the $\mathrm{NIH}$ Guide to Care and Use of Laboratory Animals and institutional guidelines.

\section{Homecage CORT in the Drinking Water}

Rats were given one bottle (1-ml graduates) fitted with a ball bearing stopper (to limit spillage) containing CORT $(300 \mu \mathrm{g} / \mathrm{ml})$ or water as the sole available fluid for 7 days ( $24 \mathrm{~h}$ daily access). CORT concentration was selected based on our previous work (Besheer et al, 2012b; Besheer et al, 2013). Rats were weighed daily and the fluid was measured and changed daily. In our laboratory, this method of CORT exposure results in heightened plasma corticosterone levels ( 400 ng/ml during the dark cycle; almost a twofold increase relative to controls). Importantly, CORT rhythmicity is maintained, albeit exaggerated (ie, heightened CORT levels during the dark cycle and lowered CORT levels during the light cycle- $\sim 30 \%$ lower than Water controls-see (Besheer et al, 2012b). Given that we have previously characterized CORT levels using this method (Besheer et al, 2012b; Besheer et al, 2013), plasma CORT analyses were not conducted in this work.

\section{Immunohistochemistry Procedure and Quantification}

Immunohistochemistry (IHC) staining and quantification procedures were similar to those we have previously described (Besheer et al, 2012a; Besheer et al, 2009; Cannady et al, 2011). Free-floating coronal sections were incubated in a rabbit anti-c-Fos antibody (1:20 000 dilution, Oncogene Research Products/Calbiochem) for $48 \mathrm{~h}$ or in a rabbit anti-mGluR5 antibody (1:1000 dilution, Millipore) for $16 \mathrm{~h}$ at $4{ }^{\circ} \mathrm{C}$ with agitation. Data (mGluR5 positive pixels/ $\mathrm{mm}^{2}$ or c-Fos positive cells $/ \mathrm{mm}^{2}$ ) were acquired from a minimum of three sections/brain region/animal, and the data were averaged to obtain a single value per subject. The brain regions examined were the nucleus accumbens (shell and core) and the dorsomedial caudate putamen all at AP $1.7-1.0 \mathrm{~mm}$.

\section{Western Blot Procedure and Quantification}

Procedures were similar to those described in (Besheer et al, 2009). Brain tissue punches (1.2 mm; UniCore, Sigma) of the nucleus accumbens were taken from coronal sliced sections (1 mm thickness). Tissue was incubated overnight at $4{ }^{\circ} \mathrm{C}$ in a polyclonal rabbit anti-mGluR5 (Millipore; 1:1000) and monoclonal mouse actin (Millipore; 1:5000) in blocking solution. Protein expression was visualized using a chemiluminescent substrate (ECL; Pierce Biotechnology, Rockford, IL) before quantification using optical density measurements (Scion Image). Data were converted to percent actin for each blot and expressed as percent control (Water group). 


\section{Alcohol Discrimination Training and Testing Procedures}

Discrimination training and testing procedures were identical to those described in detail (Besheer et al, 2012b; Besheer et al, 2009; Cannady et al, 2011). Rats were trained to discriminate a moderate dose of alcohol ( $1 \mathrm{~g} / \mathrm{kg}$, IG) from water using standard two-lever operant procedures. Testing began when the accuracy criteria were met: the percentage of appropriate lever responses before the first reinforcer and during the entire session was $>80 \%$ for at least 8 out of 10 consecutive days. For testing, cumulative dosing (Besheer et al, 2012b; Besheer et al, 2009; Cannady et al, 2011) or single alcohol dose $(1 \mathrm{~g} / \mathrm{kg})$ procedures were used as indicated in the Experiments. For the studies in Experiment 4 and 5, a baseline cumulative alcohol session was conducted before the initiation of the 7-day CORT or Water procedure (Supplementary Tables 2 and 3; Supplementary Figures 1 and 2).

\section{Cannulae Implantation Surgery, Microinjection Procedures, and Verification}

Surgical and microinjection procedures were identical to those described previously (Besheer et al, 2012a; Besheer et al, 2009; Cannady et al, 2011). Rats received bilateral implantation of 26-gauge guide cannulae (Plastics One, Roanoke, VA) aimed to terminate $2 \mathrm{~mm}$ above the nucleus accumbens core (coordinates: AP + 1.7, $\mathrm{ML}+1.5 \mathrm{~mm}$, DV $-5.5 \mathrm{~mm}$ ) as described in Paxinos and Watson (1998). Only data from rats with cannulae determined to be in the target brain regions were used in the analyses, and the sample sizes reported in the experiments are in accordance with the analyses.

\section{Experimental Procedures}

Experiment 1: Effect of CORT exposure on mGluR5 expression in the nucleus accumbens. Experimentally naive rats received CORT or water in the homecage for 7 days. Upon completion of the seventh day of CORT exposure, brain tissue was collected for the IHC $(n=8 /$ group) or western blot analysis ( $n=8-9$ /group).

Experiment 2: Effect of CORT exposure on c-Fos expression in the striatum in response to alcohol $(1 \mathrm{~g} / \mathrm{kg})$. Experimentally naive rats received CORT or water in the homecage for 7 days. Upon completion of the seventh day of exposure, rats were administered alcohol (0 (water) or $1 \mathrm{~g} / \mathrm{kg}, \mathrm{IG} ; n=6-8 /$ group/alcohol dose). Approximately $90 \mathrm{~min}$ after the injection, brain tissue was collected for c-Fos IHC.

Experiment 3: Effect of mGluR5 positive allosteric modulator on alcohol sensitivity. The effects of positive modulation of mGluR5 following systemic injection of the positive allosteric modulator 3-Cyano-N-(1,3-diphenyl-1Hpyrazol-5-yl)benzamide (CDPPB) on the discriminative stimulus effects of alcohol have not been previously determined. Therefore, the first priority was to determine whether CDPPB alone would alter the interoceptive effects of alcohol in discrimination-trained rats and which dose should be selected for Experiment 4. CDPPB $(0-40 \mathrm{mg} / \mathrm{kg}$, IP) was administered $15 \mathrm{~min}$ before the first alcohol dose of the cumulative alcohol $(0.1-1.7 \mathrm{~g} / \mathrm{kg}, \mathrm{IG})$ substitution test. Two groups of discrimination-trained rats were used for this assessment to facilitate the testing of a broad CDPPB dose range. In one group of rats $(n=13)$ a lower dose range was tested $(0,1,10 \mathrm{mg} / \mathrm{kg}$, IP), and in the other group $(n=7)$ a higher dose range was tested $(0,20,40 \mathrm{mg} / \mathrm{kg}, \mathrm{IP})$. In each group a within subject design was used and CDPPB dose order testing was randomized. One rat in the High group died during the course of the experiment (unrelated to CDPPB injection) and was not tested on the $40 \mathrm{mg} / \mathrm{kg}$ dose.

Experiment 4: Effect of mGluR5-positive allosteric modulator on alcohol sensitivity following CORT exposure. Next, we sought to determine whether positive modulation of mGluR5 could restore sensitivity to alcohol following CORT exposure. In a separate group of discrimination-trained rats, after a baseline cumulative alcohol substitution curve $(0.1,0.5,1.0,1.7 \mathrm{~g} / \mathrm{kg}, \mathrm{IG})$ was determined, homecage CORT or Water exposure ( $n=30-34$ /group) began for 7 days. Discrimination training was withheld during this time and rats remained in the homecage for the 7-day duration as we describe in Besheer et al (2012b). Upon completion of Day 7, rats received injection of $\mathrm{CDPPB}(0,10$, or $20 \mathrm{mg} / \mathrm{kg}$, IP; $n=7-12 /$ group $/ \mathrm{CDPPB}$ dose) $20 \mathrm{~min}$ before administration of alcohol ( $1 \mathrm{~g} / \mathrm{kg}$, IG). Rats were then placed in the chamber for a test session.

Experiment 5: Effect of intra-accumbens mGluR5 agonist on alcohol sensitivity following CORT exposure. The goal of this experiment was to determine whether intraaccumbens mGluR5 activation could restore sensitivity to alcohol. Discrimination-trained rats were implanted with bilateral cannulae aimed at the nucleus accumbens core. After a baseline cumulative alcohol substitution curve (0.1, $0.5,1.0,1.7 \mathrm{~g} / \mathrm{kg}, \mathrm{IG}$ ) was determined, homecage CORT or Water exposure ( $n=23-24$ /group) began for 7 days (as described in Experiment 4). Upon completion of Day 7, the mGluR5 agonist CHPG was microinfused into the nucleus accumbens $(0,5$, or $10 \mu \mathrm{g} / 0.5 \mu \mathrm{l}$ per side; $n=7-11$ /group/ CHPG dose). After the $1.5 \mathrm{~min}$ CHPG diffusion period, rats received an alcohol injection (1 g/kg, IG) and were placed in the chamber for a test session. The selection of CHPG arose out of necessity due to technical (solubility) issues. That is, we were unable to utilize the positive allosteric modulator CDPPB for brain injections and as such the orthosteric agonist for mGluR5 CHPG was used (also see Supplementary Discussion).

Next, we sought to determine whether CHPG would potentiate the interoceptive effects of a low dose of alcohol (no CORT or Water exposure in this assessment). Rats received an intra-accumbens infusion of CHPG $(0,5$, or $10 \mu \mathrm{g} / 0.5 \mu \mathrm{l}$ per side; $n=7-8 /$ group) before alcohol $(0.3 \mathrm{~g} / \mathrm{kg}, \mathrm{IG})$. This alcohol dose was selected as it induced alcohol-appropriate responding at similar levels to that observed following CORT exposure $(<40 \%)$. Some of the rats in this assessment were from the Water groups in the previous assessment and others had been used in an unrelated experiment. 


\section{Drugs}

Alcohol (95\%) was diluted in distilled water to a concentration of $20 \%(\mathrm{v} / \mathrm{v})$ and administered IG, with volumes varied by weight to obtain the desired dose. Corticosterone hemisuccinate (4-pregnen-11 $\beta, 21-D I O L-3,20-D I O N E ~ 21-$ hemisuccinate; Steraloids, Newport, RI) was dissolved in tap water by addition of $\mathrm{NaOH}$ and neutralized with $\mathrm{HCl}$, to a final pH of 7.0-7.4 (Besheer et al, 2012b; Besheer et al, 2013; Gourley and Taylor, 2009). 3-Cyano-N-(1,3-diphenyl-1Hpyrazol-5-yl)benzamide (CDPPB; Tocris) dose and pretreatment interval were chosen based on the literature (Clifton et al, 2013; Fowler et al, 2011; Gass and Olive, 2009; Stefani and Moghaddam, 2010). The half-life of the drug is $4.4 \mathrm{~h}$ (Kinney et al, 2005), and therefore suitable for the cumulative alcohol testing procedure ( $\sim 48 \mathrm{~min})$. CDPPB was suspended in $0.5 \%$ carboxymethylcellulose and injected intraperitoneally at a volume of $2 \mathrm{ml} / \mathrm{kg}$. (R,S)-2-Amino-2(2-chloro-5-hydroxyphenyl)acetic acid sodium salt (CHPG; Abcam) was dissolved in artificial CSF (aCSF), and the selected doses were based on our previous work (Besheer et al, 2009).

\section{Data Analysis}

Group differences in mGluR5 IR and protein expression were determined by $t$-test. For the c-Fos studies, each brain region was examined separately by two-way ANOVA. For the discrimination experiments, response accuracy was expressed as the percentage of alcohol-appropriate lever presses upon delivery of the first reinforcer. Response rate (responses/min) was analyzed for the entire session and served as an index of motor activity. Complete expression of the discriminative stimulus effects of alcohol (ie, full substitution) was defined as $>80 \%$ choice of the alcohol lever upon completion of the first FR10 during test sessions. If an animal did not complete an FR10 during these test sessions, then data from that animal were not included in the response accuracy analysis, but were included in the response rate analysis. One or two-way repeated measures analysis of variance (RM ANOVA) was used to analyze response accuracy and response rate data. Tukey post hoc analyses were used to explore significant interactions. Significance was declared at $P \leqslant 0.05$.

\section{RESULTS}

For Experiments 1 and 2, average daily fluid consumption and CORT dose consumed are shown in Supplementary Table 1. For Experiments 4 and 5, baseline discrimination performance and average daily fluid consumption and CORT dose consumed are shown in Supplementary Tables 2-3 and Supplementary Figures 1-2.

\section{Experiment 1: Effect of CORT Exposure on mGluR5 Expression in the Nucleus Accumbens}

Following 7 days of CORT exposure, there was a significant (twofold) reduction in mGluR5 IR in the nucleus accumbens core (Figure $1 \mathrm{a}-\mathrm{c} ; t=2.78, p=0.02$ ), but no change in the shell. No CORT-induced change was observed in the dorsomedial striatum (caudate putamen), which served as a control region given that this region has been shown to have similar mGluR5 expression as the nucleus accumbens (Besheer et al, 2009).

mGluR5 protein expression in the nucleus accumbens was also significantly reduced following CORT exposure (Figure $1 \mathrm{~d}$ and e). In brain tissue, mGluR5 is present as monomer and dimer forms and a significant reduction in mGluR5 protein expression was observed in the dimer form $(\sim 250 \mathrm{kDa} ; t=2.11, p=0.05)$, but not the monomer form $(\sim 130 \mathrm{kDa})$. This is important given that the dimer form is the active form of mGluR5 (Pin et al, 2003).

\section{Experiment 2: Effect of CORT Exposure on c-Fos Expression in the Striatum in Response to Alcohol (1 g/kg)}

Following CORT exposure, an alcohol ( $1 \mathrm{~g} / \mathrm{kg})$-induced increase in c-Fos IR was prevented in the nucleus accumbens core (Figure $2 \mathrm{a}$ and $\mathrm{b}$ ) as confirmed by a significant main effect of alcohol treatment $\left(\mathrm{F}_{(1,28)}=4.62\right.$, $p=0.04)$ and a significant alcohol dose by CORT exposure interaction $\left(\mathrm{F}_{(1,28)}=5.13, p=0.03\right)$. In the Water group, alcohol ( $1 \mathrm{~g} / \mathrm{kg}, \mathrm{IG})$ significantly increased c-Fos IR as compared with vehicle $(p<0.05)$. This alcohol-induced increase in c-Fos IR was not evident in the CORT group. No CORT- or alcohol-induced changes in c-Fos IR were observed in the nucleus accumbens shell (Figure $2 \mathrm{c}$ and $\mathrm{d}$ ) as determined from the lack of any significant main effects or interaction. Interestingly, under vehicle conditions visual inspection of Figure $2 \mathrm{c}$ appears to show a trend for increased c-Fos IR in the CORT group; however, post hoc comparisons were not prompted given the lack of any significant main effects or interaction. No CORT- or alcohol-induced changes were observed in the dorsomedial striatum (mean \pm SEM c-Fos positive cells $/ \mathrm{mm}^{2}$-Water/ Vehicle: $8.53 \pm 2.06$; CORT/Vehicle: $11.68 \pm 2.21$; Water/ Alcohol: $10.03 \pm 2.06$; CORT/Alcohol: $10.38 \pm 2.06$ ). Together these data show that a period of repeated and heightened elevations in the stress hormone CORT prevents an increase in neural activity in the nucleus accumbens core in response to a moderate alcohol dose $(1 \mathrm{~g} / \mathrm{kg}, \mathrm{IG})$.

\section{Experiment 3: Effect of mGluR5-Positive Allosteric Modulator on Alcohol Sensitivity}

CDPPB did not potentiate or blunt the discriminative stimulus effects of alcohol under 'normal' conditions (eg, no CORT exposure in this experiment). The two different groups of rats used for this assessment did not differ under vehicle conditions (see Supplementary Results), and, for ease of presentation, they are graphically presented together (Figure 3). In the Low and High groups, alcohol-appropriate responding increased with alcohol dose (Low $\left(\mathrm{F}_{(3,36)}=36.22, p<0.001\right.$; High $\left.\left(\mathrm{F}_{(3,15)}=72.32, p<0.001\right)\right)$, with no significant main effects of CDPPB dose or interactions observed, indicating that CDPPB did not alter the discriminative stimulus effects of alcohol (Figure 3a). In both the Low and High groups, response rate decreased with increasing alcohol doses (Low $\left(\mathrm{F}_{(3,36)}=6.76, p<0.001\right.$; High $\left.\mathrm{F}_{(3,15)}=5.94, p=0.007\right)$; Figure $\left.3 \mathrm{~b}\right)$. In the High group, a significant main effect of CDPPB dose was observed $\left(\mathrm{F}_{(2,10)}=9.58, p=0.005\right)$, with a significant reduction in 
a

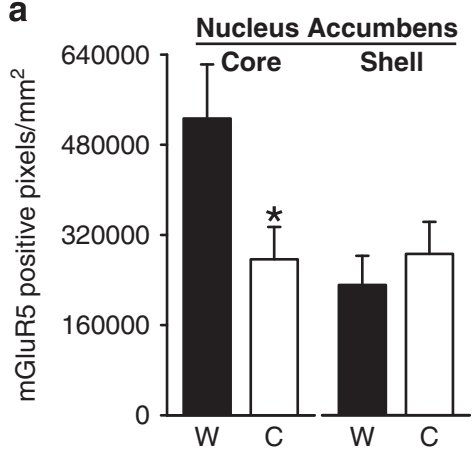

b

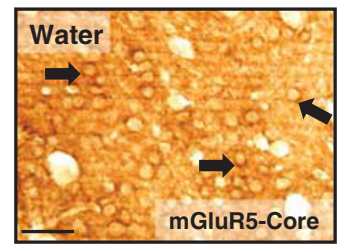

c

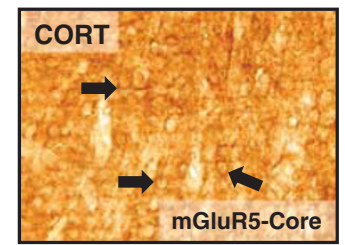

d

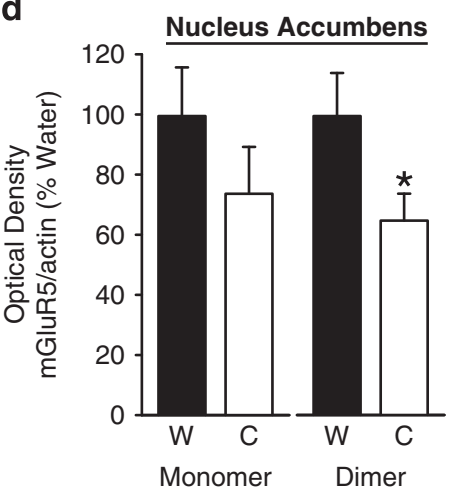

e

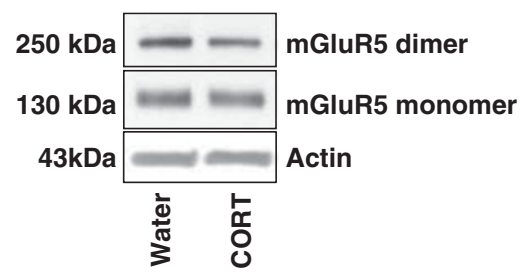

Figure I Significant reductions in mGluR5 expression in the nucleus accumbens following CORT exposure (7 days). (a) Following CORT exposure (7 days) a significant reduction in mGluR5 immunoreactivity (IR) is evident in the core, but not the shell of the nucleus accumbens. No change in mGluR5 IR in the dorsomedial (DM) caudate putamen was observed (CPu). (b, c) Representative photomicrographs $(\times 40)$ showing cytological expression of mGlu5 receptors in the core for the Water and CORT groups. Arrows identify areas of dense mGlu5 receptor immunoreactivity. Scale bar represents 50 microns. (d) Decreased mGluR5 dimer protein expression $(250 \mathrm{kDa})$ was observed in the nucleus accumbens core as confirmed by western blot analysis following CORT exposure (7 days). Monomer ( $30 \mathrm{kDa}$ ) expression was unaltered. (e) Representative western blots showing decreased mGluR5 expression in the mGluR5 dimer (right) in the CORT group. *Signifies differences from Water. Values on graphs represent mean \pm SEM.

a
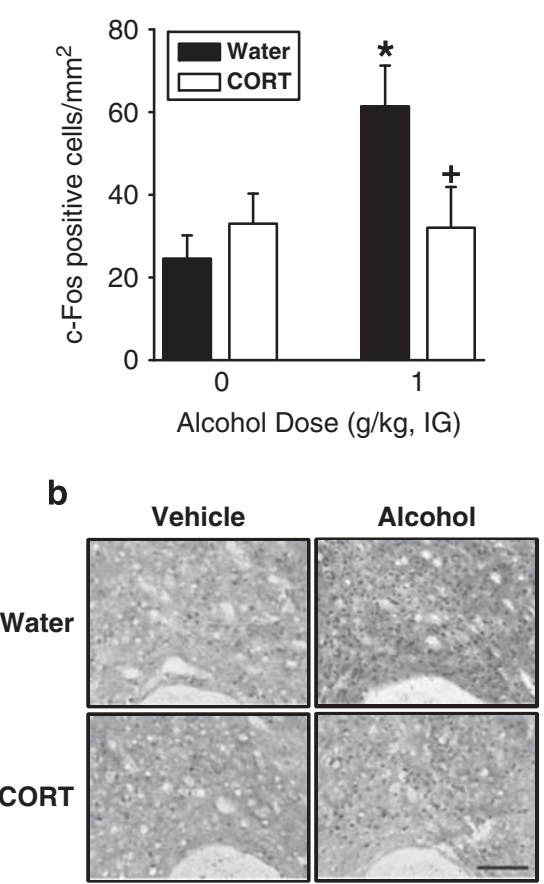

\section{Nucleus Accumbens Shell}

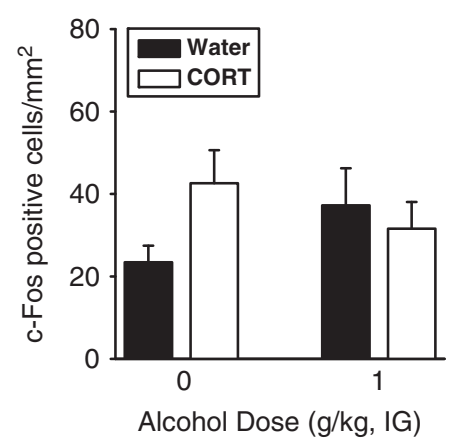

d

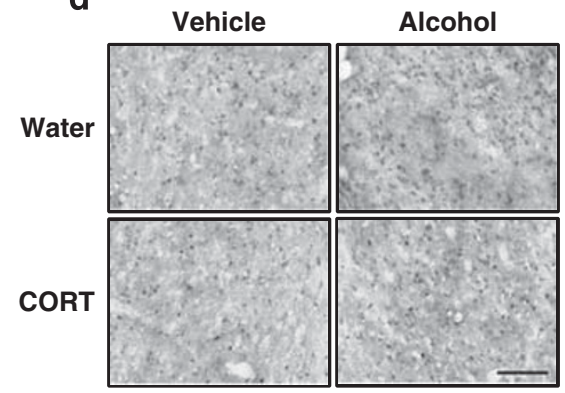

Figure 2 Blunted neural activity in the nucleus accumbens core in response to a moderate alcohol dose ( I g/kg, IG) following CORT exposure (7 days). (a) Vehicle (water, IG) administration did not change c-Fos immunoreactivity (IR) between the Water and CORT-exposed groups. Alcohol (I g/kg, IG) induced a significant increase in c-Fos immunoreactivity $(\mathbb{R})$ in the nucleus accumbens core in the Water group, which was not evident in CORT-exposed group $(n=8)$. (b) Representative pictographs $(\times 20)$ showing c-Fos IR in the nucleus accumbens core in the Water and CORT groups following vehicle (water, IG) and alcohol (I g/kg, IG). Scale bar represents 100 microns. (c) No significant CORT- or alcohol-induced changes in c-Fos IR in the nucleus accumbens shell $(n=6-8)$ were observed. (d) Representative pictographs $(\times 20)$ showing c-Fos IR in the medial nucleus accumbens shell in the Water and CORT groups following vehicle (water, IG) and alcohol $(\mathrm{I} g / \mathrm{kg}, \mathrm{IG})$. Scale bar represents 100 microns. *Signifies difference from Vehicle. ${ }^{+}$Signifies difference from Water. Values on graphs represent mean \pm SEM. 

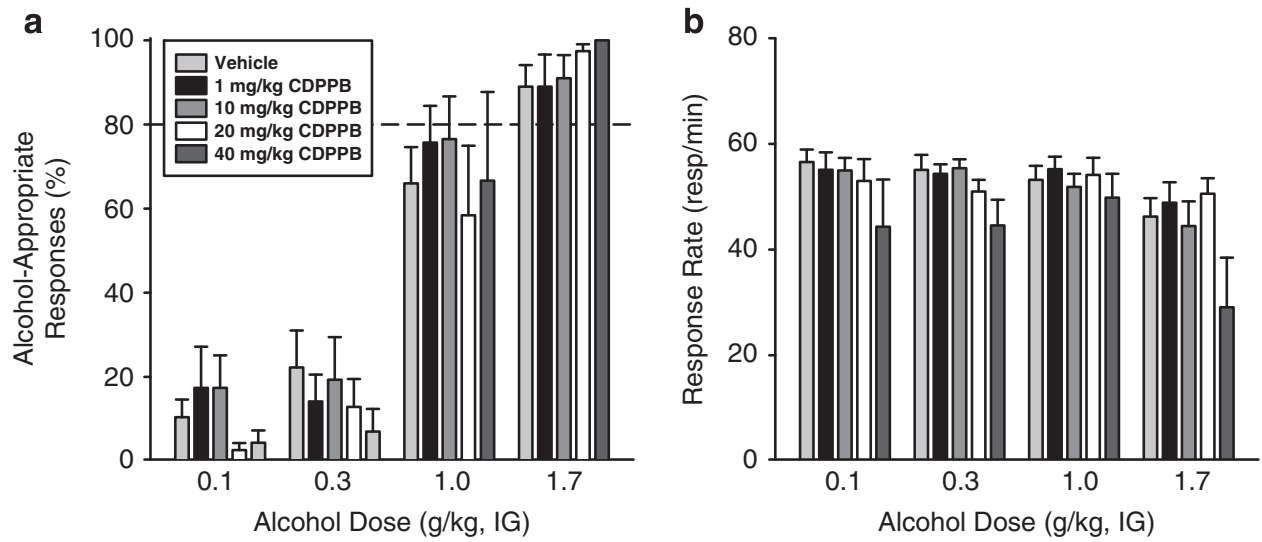

Figure 3 Positive allosteric modulation of mGluR5 does not alter the interoceptive effects of alcohol. (a) Pretreatment with CDPPB a positive allosteric modulator of mGluR5 administered IP did not alter the discriminative stimulus effects of alcohol, but (b) produced an overall reduction in response rate. These graphs represent the data from two separate assessments in which one group of rats was tested on $0,1,10 \mathrm{mg} / \mathrm{kg}$ CDPPB $(n=13)$ and the other was tested on $0,20,40 \mathrm{mg} / \mathrm{kg}(n=7)$. Therefore, the data points for the vehicle condition on the graph represent a combination of the two groups ( $n=20)$. Data from the two groups were analyzed separately but are graphically depicted together for ease of presentation. Horizontal dashed line (>80\%) represents full expression of the discriminative stimulus effects of alcohol. Values on graphs represent mean \pm SEM.

response rate after pretreatment with the highest dose $(40 \mathrm{mg} / \mathrm{kg})$ relative to the vehicle $(p<0.05)$. Given the overall lack of CDPPB effect on modulating the discriminative stimulus effects of alcohol, the two highest doses that did not alter response rate $(10$ and $20 \mathrm{mg} / \mathrm{kg})$ were selected for testing in Experiment 4.

\section{Experiment 4: Effect of mGluR5-Positive Allosteric Modulator on Alcohol Sensitivity Following CORT Exposure}

The goal of this assessment was to determine whether pretreatment with CDPBB before the alcohol training dose $(1 \mathrm{~g} / \mathrm{kg}, \quad \mathrm{G})$ would restore a CORT exposure-induced reduction in sensitivity to alcohol. Following CORT exposure, a significant overall reduction in alcohol-appropriate responses was observed $\left(\mathrm{F}_{(1,58)}=8.95, p=0.004\right.$; Figure $4 \mathrm{a})$. There was a trend for a significant main effect of CDPPB dose $\left(\mathrm{F}_{(2,58)}=2.90, p=0.06\right)$. The exposure by CDPPB dose interaction was significant $\left(\mathrm{F}_{(2,58)}=5.32\right.$, $p=0.008$ ), and post hoc comparisons showed a significant reduction in alcohol-appropriate responding following vehicle pretreatment in the CORT group relative to the Water group $(p<0.001)$. This data pattern is evidence of decreased sensitivity to the interoceptive effects of alcohol following CORT exposure, consistent with previous work (Besheer et al, 2012b). Importantly, CDPPB increased sensitivity to alcohol in the CORT group, with significantly greater alcohol-appropriate responding with both doses of CDPPB relative to vehicle $(p$ 's $<0.01)$, with the highest CDPPB dose $(20 \mathrm{mg} / \mathrm{kg})$ fully restoring sensitivity to the alcohol training dose $(1 \mathrm{~g} / \mathrm{kg})$. Response rate was not altered by CORT exposure or CDPPB pretreatment (Figure 4b). Importantly, restored sensitivity to alcohol in the CORT group occurred at CDPPB doses that did not potentiate the discriminative stimulus effects of alcohol when tested under 'normal'/control conditions as determined in Experiment 3.

\section{Experiment 5: Effect of Intra-Accumbens mGluR5 Agonist on Alcohol Sensitivity Following CORT Exposure}

To determine the functional involvement of intra-accumbens mGluR5 and whether activation of these receptors would restore sensitivity to alcohol following CORT exposure, rats received intra-accumbens injection of CHPG before the alcohol training dose $(1 \mathrm{~g} / \mathrm{kg}, \mathrm{IG})$. There was a significant main effect of group $\left(\mathrm{F}_{(1,41)}=6.11, p=0.02\right)$, a significant main effect of pretreatment injection (aCSF or CHPG; $\mathrm{F}_{(2,41)}=7.11, p=0.002$ ), and a significant interaction $\left(\mathrm{F}_{(2,41)}=7.27, p=0.002\right.$; Figure $\left.5 \mathrm{a}\right)$, with a significant decrease in alcohol-appropriate responding in the CORT group relative to the Water control group following intraaccumbens aCSF injection $(p<0.001)$. This data pattern confirms decreased sensitivity to the interoceptive effects of alcohol following CORT exposure consistent with vehicle administration in Experiment 4 and Besheer et al, 2012b. Further, sensitivity to alcohol was restored by intraaccumbens pretreatment of both CHPG doses $(p<0.001)$. Analysis of response rate showed a significant main effect of group $\left(\mathrm{F}_{(1,41)}=5.75, p=0.02\right)$, with the CORT group showing slightly lower response rates than the Water group overall ( $p=0.02$; Figure $5 b)$.

In a follow-up experiment, we determined that intraaccumbens administration of CHPG at the doses found to restore sensitivity to alcohol did not potentiate the discriminative stimulus effects of a low alcohol dose $(0.3 \mathrm{~g} / \mathrm{kg}, \quad \mathrm{IG})$ under 'normal' conditions (ie, absence of CORT; see Supplementary Results). Therefore, the lack of potentiation of this dose of alcohol suggests that at these CHPG doses (1) CHPG is not pharmacologically potentiating the effects of a low alcohol dose, and (2) CHPG alone is likely not having alcohol-like effects. Therefore, we conclude that sensitivity to alcohol following CORT exposure was restored at CHPG doses that did not potentiate the interoceptive effects of a low dose of alcohol. 


\section{DISCUSSION}

The results of the present study show neuroadaptations in the ventral striatum as a consequence of a period of exposure to the stress hormone CORT. Decreased mGluR5 expression in the hippocampus has been observed following chronic CORT injections (Iyo et al, 2010) and following chronic mild stress exposure (Wieronska et al, 2001). However, this is the first study to show decreased mGluR5 expression in the nucleus accumbens following CORT exposure, and this finding adds to the growing literature identifying neuroadaptations within this brain region as a consequence of CORT or repeated stressor exposure (Campioni et al, 2009; Gourley and Taylor, 2009; Lemos et al, 2012; Morales-Medina et al, 2009; Perrotti et al, 2004; Shoji and Mizoguchi, 2010; Tidey and Miczek, 1997). Importantly, the presence of CORT-induced neuroadaptations in mGluR5, as we show here, potentially has broad
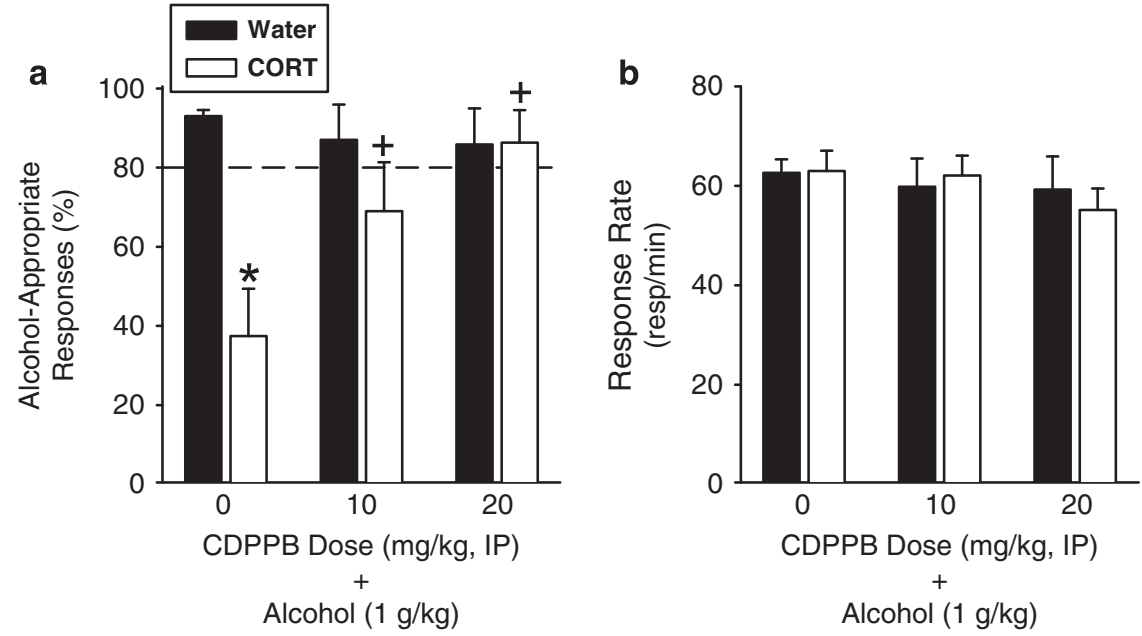

Figure 4 Following CORT exposure, positive allosteric modulation of mGluR5 restores sensitivity to the interoceptive effects of alcohol. (a) CORT exposure (7 days) prevented the full expression of the discriminative stimulus effects of alcohol $(\mathrm{I} / \mathrm{g} / \mathrm{kg})$ following vehicle $(0)$ pretreatment. In contrast, pretreatment with CDPPB a positive allosteric modulator of mGluR5 restored sensitivity to alcohol ( $n=7-12 / g r o u p / C D P P B ~ d o s e)$. (b) Response rate was not affected by CORT exposure or CDPPB pretreatment. *Signifies differences from Water; ${ }^{+}$signifies difference from 0 (vehicle-CORT group; Tukey, $p<0.05)$. Values on graphs represent mean \pm SEM.
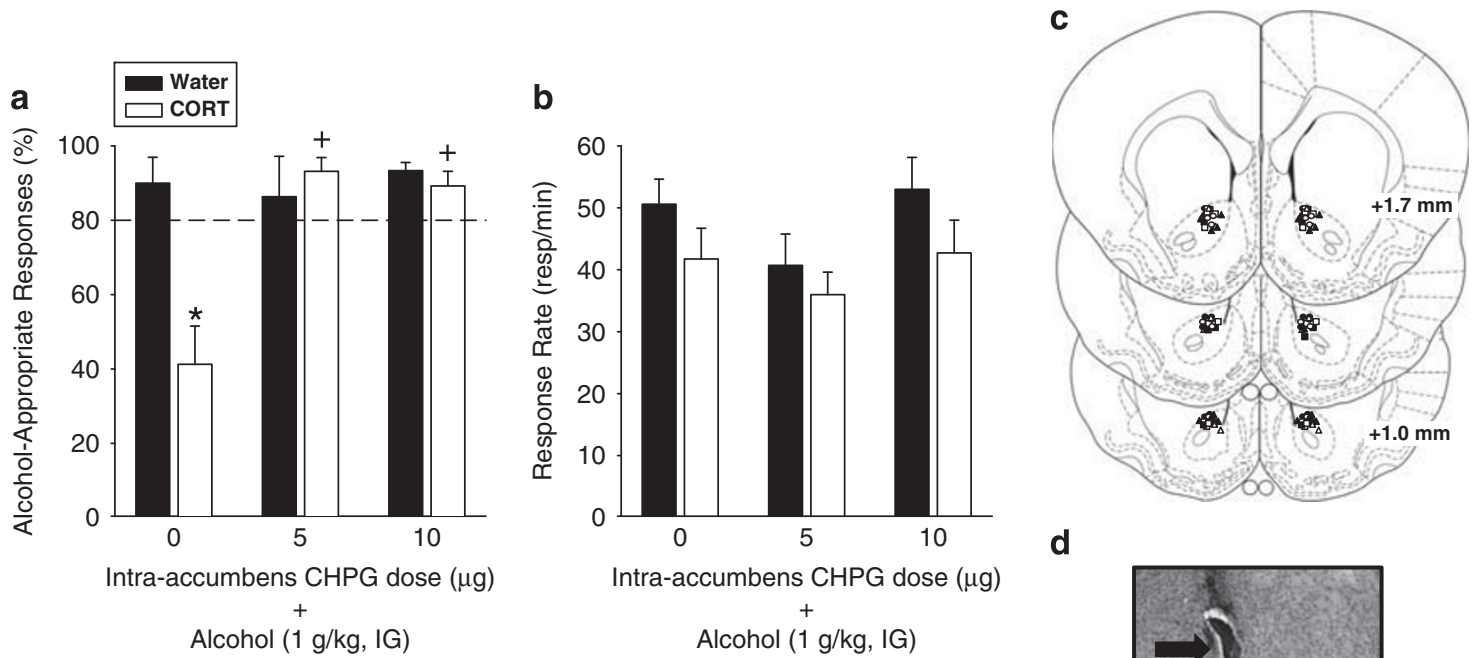

d

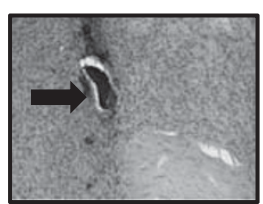

Figure 5 mGluR5 activation in the nucleus accumbens restores sensitivity to alcohol following CORT exposure. (a) Site specific activation of mGluR5 in the nucleus accumbens core by CHPG (5 and $10 \mu \mathrm{g} /$ side) restored sensitivity to alcohol $(\mathrm{I} / \mathrm{g} / \mathrm{kg}, \mathrm{IG})$ following CORT exposure, as indicated by similar levels of alcohol-appropriate responses following CHPG pretreatment between the CORT and Water groups ( $n=7-$ II/group/CHPG dose), and significantly greater alcohol-appropriate responses in the CHPG-treated CORT groups than the vehicle-treated CORT group. *Signifies significant difference from Water. ${ }^{+}$signifies significant difference from 0 (aCSF-CORT group) Tukey, $p<0.05$ ). (b) There was a significant overall reduction in response rate following CORT exposure. (c) Nucleus accumbens (core) bilateral injector tip placements from individual rats with accurate placements. The groups are represented by the following symbols: Circles (Vehicle), triangles ( $5 \mu \mathrm{g}$ CHPG), squares ( $10 \mu \mathrm{g}$ CHPG); filled and unfilled symbols are placements from the Water and CORT groups, respectively. (d) A corresponding photomicrograph showing an injector tip (arrow). Values on graphs represent mean \pm SEM. 
implications and may be relevant to better understanding some mental health disorders (ie, depression, post-traumatic stress disorder), specifically as chronic CORT exposure is commonly used to induce various behavioral aspects of these disorders (Ardayfio and Kim, 2006; Gourley and Taylor, 2009; Hache et al, 2012; Rainer et al, 2011). Further, this is especially relevant given that pharmacological manipulation of mGluR5 and other mGluRs is of interest as potential therapeutics for the treatment of neuropsychiatric disorders (Krystal et al, 2010; Witkin et al, 2007).

The findings of decreased accumbal mGluR5 expression following CORT exposure is consistent with other work showing decreased mGluR5 expression in the hippocampus following 21 days of CORT injections (Iyo et al, 2010). Given the absence of evidence, to date, to suggest that glucocorticoids directly regulate mGluR5, one explanation for the decrease in mGluR5 expression may be related to a compensatory neuroadaptation as a consequence of increased glutamate levels (ie, chronic receptor activation by the endogenous ligand; Iyo et al, 2010; Shaffer et al, 2010). Indeed, enhanced extracellular glutamate levels have been reported in the striatum following acute stressor exposure (Moghaddam, 1993; Sequeira-Cordero et al, 2014), and elevations in basal extracellular glutamate levels in the nucleus accumbens core, but not shell, have been reported 21 days after a stressor exposure (Garcia-Keller et al, 2013). Further, a hyperglutamatergic state in the nucleus accumbens is also suggested from electrophysiological assessments following CORT administration (2 day) or chronic unpredictable stress (Campioni et al, 2009; Wang et al, 2010). While the focus of the present work is on mGluR5 in the nucleus accumbens, it is important to consider that exposure to heightened CORT levels undoubtedly has widespread consequences throughout the brain (Herman et al, 2005; McEwen, 2007). Therefore, another possibility for the decrease in accumbal mGluR5 may be related to adaptations in neurocircuitry that involve and impact the nucleus accumbens. In support of this explanation is the finding that the IHC analysis showed a core-specific reduction in mGluR5 IR. The core and shell of the nucleus accumbens receive anatomically distinct inputs, including dense glutamatergic projections from the hippocampus and the prefrontal cortex (Basar et al, 2010; Britt et al, 2012; Cotman and Monaghan, 1986; Groenewegen and Trimble, 2007). Interestingly, glucocorticoid-induced adaptations in glutamatergic neurotransmission have been well documented in these brain regions (Chaouloff and Groc, 2011; Joca et al, 2007; Popoli et al, 2012). For example, exposure to chronic stress or systemic CORT can enhance glutamate release from the hippocampus (Bagley and Moghaddam, 1997; Skorzewska et al, 2007; Venero and Borrell, 1999). As a consequence, it is possible that such presynaptic changes could impact excitatory drive into the nucleus accumbens, perhaps leading to differential core $v s$ shell neuroadaptations. While the goal of the present work was to assess the functional role of the CORT-induced change in accumbal mGluR5 on interoceptive sensitivity to alcohol, clearly, it will be necessary for future studies to delineate the cause(s) for the decrease in mGluR5 following CORT exposure. In addition, it will be of interest to examine whether Homer, a scaffold protein for mGluR5 (Brakeman et al, 1997), is also altered, given that this family of proteins regulates signal transduction and trafficking of mGluR5 (Xiao et al, 2000). Examination of Homer 1 and 2 isoforms, which are highly expressed in corticolimbic circuits and implicated in modulating drug-induced neuroplasticity and possibly some neuropsychiatric disorders (Szumlinski et al, 2008), may be of particular interest to inform the functional implications of the reduction in mGluR5 expression reported in the present work.

Neural response to alcohol was also altered in the nucleus accumbens as a consequence of CORT exposure. That is, in response to alcohol ( $1 \mathrm{~g} / \mathrm{kg}, \mathrm{IG})$ a threefold increase in c-Fos IR was observed in the nucleus accumbens core. This subregional dissociation (core $v s$ shell) in response to alcohol is consistent with previous findings (Bachtell et al, 1999; Besheer et al, 2008); however, this is the first study to show blunted accumbal response to alcohol as a consequence of stress hormone exposure. Interestingly, based on our previous characterization of this CORT exposure procedure, circulating plasma CORT levels in the CORT group are predicted to be lower than the Water group at the time of alcohol injection (Besheer et al, 2012b; Besheer et al, 2013). Previous work argues against a direct relation between reduced circulating plasma CORT levels at time of testing and sensitivity to the interoceptive effects of alcohol (Besheer et al, 2012b); however, it is possible that the blunted accumbal (core) c-Fos response to alcohol in the CORT group may be directly related to decreased plasma CORT level. Indeed, there is support for a positive association between plasma CORT levels and c-Fos expression in the nucleus accumbens in response to drug administration (Badiani et al, 1998; Tomita et al, 2013). Interestingly, under control conditions (ie, after water administration) c-Fos IR in the core does not differ between the CORT and Water groups; however, we cannot discount the possibility that following alcohol administration a relation between plasma CORT levels and c-Fos expression may emerge. Nonetheless, given the known role of the nucleus accumbens in modulating the interoceptive effects of alcohol, these findings suggest that decreased sensitivity to the interoceptive effects of alcohol following CORT exposure may be functionally related to this blunted brain regional response to alcohol. Moreover, while we cannot conclude that the blunted c-Fos response to alcohol is related to the change in mGluR5 expression, it is interesting to note the similarity in subregional specificity (ie, core $v s$ shell).

To address the functional implications of the CORTinduced change in mGluR5 in relation to the interoceptive effects of alcohol, the effects of pharmacological positive modulation (CDPPB) and activation (CHPG) of mGluR5 were assessed. Pretreatment with systemically administered CDPPB dose-dependently restored sensitivity to alcohol ( $1 \mathrm{~g} / \mathrm{kg}, \mathrm{IG})$ following CORT exposure. This was likely due, in part, to the contribution of intra-accumbens mGluR5 as site-specific pretreatment with CHPG restored sensitivity to alcohol under the same conditions. Interestingly, restored sensitivity to alcohol occurred at CDPPB and CHPG doses that did not potentiate the interoceptive effects of alcohol under 'normal'/control conditions. Previous work has shown that activity at intra-accumbens mGluR 5 is necessary and sufficient for the expression of the interoceptive effects 
of alcohol (Besheer et al, 2009). Therefore, a possible interpretation of the present findings is that animals show increased sensitivity to mGluR5 activation following CORT exposure. Alternatively, pretreatment with the mGluR5 compounds may pharmacologically compensate for the functional downregulation of mGluR5. Lastly, positive allosteric modulators of mGluR5 are emerging as pharmacological tools to enhance cognitive function and reverse memory impairments (Fowler et al, 2013; Gass and Olive, 2009; Liu et al, 2008; Reichel et al, 2011; Stefani and Moghaddam, 2010; Vales et al, 2010). However, a memory enhancement explanation may be less tenable given that we have previously confirmed that the reduction in alcoholappropriate responding following CORT exposure is unrelated to a memory or performance deficit (Besheer et al, 2012b). In summary, these data suggest that activation of mGluR5 may have alleviated the functional impact of the CORT-induced downregulation of mGluR5 in relation to sensitivity to alcohol.

An unexpected finding from the present work was that systemic CDPPB pretreatment did not alter the discriminative stimulus effects of alcohol under 'normal' conditions (Experiment 3). In light of our previous findings in which an mGluR5 agonist injected into the nucleus accumbens potentiated the interoceptive effects of a low alcohol dose $(0.5 \mathrm{~g} / \mathrm{kg})$ under control conditions (Besheer et al, 2009), we hypothesized that systemically administered CDPPB would potentiate the discriminative stimulus effects of alcohol under 'normal' conditions. The CDPPB dose range tested is considered to be moderate (see Stauffer, 2011) and was selected based on pilot experiments in our lab and previously published work (Clifton et al, 2013; Fowler et al, 2011; Gass and Olive, 2009; Stefani and Moghaddam, 2010). Therefore, there is strong possibility that a higher dose range may have potentiated the interoceptive effects of alcohol, and this would need to be examined before concluding that positive allosteric modulation of mGluR5 does not potentiate the interoceptive effects of alcohol under basal conditions.

In conclusion, decreased sensitivity to the interoceptive effects of alcohol following prolonged stress hormone exposure is linked to neuroadaptations in the ventral striatum, including decreased mGluR5 expression and blunted neuronal response to alcohol. Further, sensitivity to a moderate alcohol dose $(1 \mathrm{~g} / \mathrm{kg})$ can be restored by pharmacological activation of mGluR5, perhaps suggesting alleviation, in part, of the functional consequence of downregulated mGluR5. Importantly, alcohol is a complex stimulus cue and different receptor systems modulate sensitivity to different alcohol doses (eg, high vs low; cf, Grant, 1999; Stolerman et al, 2011). Therefore, it will be important to extend the present findings to higher alcohol training doses. While it is difficult to determine the direct contribution of the interoceptive effects of alcohol to alcohol drinking, altered sensitivity to the subjective/ interoceptive effects of alcohol has been presented as a possible behavioral mechanism for escalated alcohol drinking during episodes of heightened elevations in glucocorticoid levels, such as stress (Besheer et al, 2012b; Besheer et al, 2013; Childs et al, 2011; Soderpalm and de Wit, 2002). That is, during these episodes, an individual may consume more alcohol to achieve the desired interoceptive effects.
Therefore, understanding the functional impact of neuroadaptations that occur as a consequence of episodes of chronic elevations in glucocorticoid levels may enrich our understanding of altered subjective sensitivity to alcohol during stressful episodes.

\section{FUNDING AND DISCLOSURE}

This work was supported, in part, by funds from the National Institutes of Health AA019682 (JB), AA020914 (JB), ABMRF/The Foundation for Alcohol Research (JB), and by the Bowles Center for Alcohol Studies. The authors declare no conflict of interest.

\section{ACKNOWLEDGEMENTS}

We thank Andrew Whang and Maria Masciello for help with various aspects of behavioral studies.

\section{REFERENCES}

Ardayfio P, Kim KS (2006). Anxiogenic-like effect of chronic corticosterone in the light-dark emergence task in mice. Behav Neurosci 120: 249-256.

Bachtell RK, Wang YM, Freeman P, Risinger FO, Ryabinin AE (1999). Alcohol drinking produces brain region-selective changes in expression of inducible transcription factors. Brain Res 847: 157-165.

Badiani A, Oates MM, Day HE, Watson SJ, Akil H, Robinson TE (1998). Amphetamine-induced behavior, dopamine release, and c-fos mRNA expression: modulation by environmental novelty. J Neurosci 18: 10579-10593.

Bagley J, Moghaddam B (1997). Temporal dynamics of glutamate efflux in the prefrontal cortex and in the hippocampus following repeated stress: effects of pretreatment with saline or diazepam. Neuroscience 77: 65-73.

Banks ML, Czoty PW, Nader MA (2007). The influence of reinforcing effects of cocaine on cocaine-induced increases in extinguished responding in cynomolgus monkeys. Psychopharmacology 192: 449-456.

Basar K, Sesia T, Groenewegen H, Steinbusch HW, VisserVandewalle V, Temel Y (2010). Nucleus accumbens and impulsivity. Prog Neurobiol 92: 533-557.

Becker HC, Lopez MF, Doremus-Fitzwater TL (2011). Effects of stress on alcohol drinking: a review of animal studies. Psychopharmacology 218: 131-156.

Besheer J, Fisher KR, Cannady R, Grondin JJ, Hodge CW (2012a). Intra-amygdala inhibition of $\operatorname{ERK}(1 / 2)$ potentiates the discriminative stimulus effects of alcohol. Behav Brain Res 228: 398-405.

Besheer J, Fisher KR, Grondin JJ, Cannady R, Hodge CW (2012b). The effects of repeated corticosterone exposure on the interoceptive effects of alcohol in rats. Psychopharmacology 220: 809-822.

Besheer J, Fisher KR, Lindsay TG, Cannady R (2013). Transient increase in alcohol self-administration following a period of chronic exposure to corticosterone. Neuropharmacology 72: 139-147.

Besheer J, Grondin JJ, Cannady R, Sharko AC, Faccidomo S, Hodge CW (2010). Metabotropic glutamate receptor 5 activity in the nucleus accumbens is required for the maintenance of ethanol self-administration in a rat genetic model of high alcohol intake. Biol Psychiatry 67: 812-822.

Besheer J, Grondin JJ, Salling MC, Spanos M, Stevenson RA, Hodge CW (2009). Interoceptive effects of alcohol require mGlu5 
receptor activity in the nucleus accumbens. J Neurosci 29: 9582-9591.

Besheer J, Hodge CW (2005). Pharmacological and anatomical evidence for an interaction between mGluR5- and GABA(A) alpha1-containing receptors in the discriminative stimulus effects of ethanol. Neuropsychopharmacology 30: 747-757.

Besheer J, Schroeder JP, Stevenson RA, Hodge CW (2008). Ethanol-induced alterations of c-Fos immunoreactivity in specific limbic brain regions following ethanol discrimination training. Brain Res 1232: 124-131.

Besheer J, Stevenson RA, Hodge CW (2006). mGlu5 receptors are involved in the discriminative stimulus effects of self-administered ethanol in rats. Eur J Pharmacol 551: 71-75.

Brakeman PR, Lanahan AA, O’Brien R, Roche K, Barnes CA, Huganir RL et al (1997). Homer: a protein that selectively binds metabotropic glutamate receptors. Nature 386: 284-288.

Britt JP, Benaliouad F, McDevitt RA, Stuber GD, Wise RA, Bonci A (2012). Synaptic and behavioral profile of multiple glutamatergic inputs to the nucleus accumbens. Neuron 76: 790-803.

Campioni MR, Xu M, McGehee DS (2009). Stress-induced changes in nucleus accumbens glutamate synaptic plasticity. J Neurophysiol 101: 3192-3198.

Cannady R, Grondin JJ, Fisher KR, Hodge CW, Besheer J (2011). Activation of Group II metabotropic glutamate receptors inhibits the discriminative stimulus effects of alcohol via selective activity within the amygdala. Neuropsychopharmacology 36: 2328-2338.

Chaouloff F, Groc L (2011). Temporal modulation of hippocampal excitatory transmission by corticosteroids and stress. Front Neuroendocrinol 32: 25-42.

Childs E, O'Connor S, de Wit H (2011). Bidirectional interactions between acute psychosocial stress and acute intravenous alcohol in healthy men. Alcohol Clin Exp Res 35: 1794-1803.

Clifton NE, Morisot N, Girardon S, Millan MJ, Loiseau F (2013). Enhancement of social novelty discrimination by positive allosteric modulators at metabotropic glutamate 5 receptors: adolescent administration prevents adult-onset deficits induced by neonatal treatment with phencyclidine. Psychopharmacology 225: 579-594.

Cotman CW, Monaghan DT (1986). Anatomical organization of excitatory amino acid receptors and their properties. Adv Exp Med Biol 203: 237-252.

Cozzoli DK, Courson J, Caruana AL, Miller BW, Greentree DI, Thompson $\mathrm{AB}$ et al (2012). Nucleus accumbens mGluR5associated signaling regulates binge alcohol drinking under drinking-in-the-dark procedures. Alcoholism, Clin Exp Res 36: 1623-1633.

D'Ascenzo M, Podda MV, Fellin T, Azzena GB, Haydon P, Grassi C (2009). Activation of mGluR5 induces spike afterdepolarization and enhanced excitability in medium spiny neurons of the nucleus accumbens by modulating persistent $\mathrm{Na}+$ currents. J Physiol 587(Pt 13): 3233-3250.

D’Souza MS, Markou A (2011). Metabotropic glutamate receptor 5 antagonist 2-methyl-6-(phenylethynyl)pyridine (MPEP) microinfusions into the nucleus accumbens shell or ventral tegmental area attenuate the reinforcing effects of nicotine in rats. Neuropharmacology 61: 1399-1405.

Fowler SW, Ramsey AK, Walker JM, Serfozo P, Olive MF, Schachtman TR et al (2011). Functional interaction of mGlu5 and NMDA receptors in aversive learning in rats. Neurobiol Learn Mem 95: 73-79.

Fowler SW, Walker JM, Klakotskaia D, Will MJ, Serfozo P, Simonyi A et al (2013). Effects of a metabotropic glutamate receptor 5 positive allosteric modulator, CDPPB, on spatial learning task performance in rodents. Neurobiol Learn Mem 99: 25-31.

Friedman DP, Aggleton JP, Saunders RC (2002). Comparison of hippocampal, amygdala, and perirhinal projections to the nucleus accumbens: combined anterograde and retrograde tracing study in the Macaque brain. J Comp Neurol 450: 345-365.

Garcia-Keller C, Martinez SA, Esparza MA, Bollati F, Kalivas PW, Cancela LM (2013). Cross-sensitization between cocaine and acute restraint stress is associated with sensitized dopamine but not glutamate release in the nucleus accumbens. Eur J Neurosci 37: 982-995.

Gass JT, Olive MF (2009). Positive allosteric modulation of mGluR5 receptors facilitates extinction of a cocaine contextual memory. Biol Psychiatry 65: 717-720.

Gourley SL, Taylor JR (2009). Recapitulation and reversal of a persistent depression-like syndrome in rodents. Curr Protoc Neurosci Chapter 9: Unit 932.

Grant KA (1999). Strategies for understanding the pharmacological effects of ethanol with drug discrimination procedures. Pharmacol Biochem Behav 64: 261-267.

Groenewegen HJ, Trimble M (2007). The ventral striatum as an interface between the limbic and motor systems. CNS Spectr 12: 887-892.

Hache G, Guiard BP, Le Dantec Y, Orvoen S, David DJ, Gardier AM et al (2012). Antinociceptive effects of fluoxetine in a mouse model of anxiety/depression. Neuroreport 23: 525-529.

Herman JP (1993). Regulation of adrenocorticosteroid receptor mRNA expression in the central nervous system. Cell Mol Neurobiol 13: 349-372.

Herman JP, Ostrander MM, Mueller NK, Figueiredo H (2005). Limbic system mechanisms of stress regulation: hypothalamopituitary-adrenocortical axis. Prog Neuropsychopharmacol Biol Psychiatry 29: 1201-1213.

Huang CC, Hsu KS (2012). Activation of NMDA receptors reduces metabotropic glutamate receptor-induced long-term depression in the nucleus accumbens via a CaMKII-dependent mechanism. Neuropharmacology 63: 1298-1307.

Iyo AH, Feyissa AM, Chandran A, Austin MC, Regunathan S, Karolewicz B (2010). Chronic corticosterone administration down-regulates metabotropic glutamate receptor 5 protein expression in the rat hippocampus. Neuroscience 169: $1567-1574$.

Joca SR, Ferreira FR, Guimaraes FS (2007). Modulation of stress consequences by hippocampal monoaminergic, glutamatergic and nitrergic neurotransmitter systems. Stress 10: 227-249.

Kinney GG, O'Brien JA, Lemaire W, Burno M, Bickel DJ, Clements MK et al (2005). A novel selective positive allosteric modulator of metabotropic glutamate receptor subtype 5 has in vivo activity and antipsychotic-like effects in rat behavioral models. $J$ Pharmacol Exp Ther 313: 199-206.

Krystal JH, Mathew SJ, D’Souza DC, Garakani A, Gunduz-Bruce H, Charney DS (2010). Potential psychiatric applications of metabotropic glutamate receptor agonists and antagonists. CNS Drugs 24: 669-693.

Lemos JC, Wanat MJ, Smith JS, Reyes BA, Hollon NG, Van Bockstaele EJ et al (2012). Severe stress switches CRF action in the nucleus accumbens from appetitive to aversive. Nature 490: 402-406.

Liu F, Grauer S, Kelley C, Navarra R, Graf R, Zhang G et al (2008). ADX47273 [S-(4-fluoro-phenyl)-\{3-[3-(4-fluoro-phenyl)-[1,2,4]oxadiazol-5-yl]-piperidin-1- yl\}-methanone]: a novel metabotropic glutamate receptor 5-selective positive allosteric modulator with preclinical antipsychotic-like and procognitive activities. J Pharmacol Exper Ther 327: 827-839.

McEwen BS (2007). Physiology and neurobiology of stress and adaptation: central role of the brain. Physiol Rev 87: 873-904.

McEwen BS, De Kloet ER, Rostene W (1986). Adrenal steroid receptors and actions in the nervous system. Physiol Rev 66: 1121-1188.

Mihindou C, Vouillac C, Koob GF, Ahmed SH (2011). Preclinical validation of a novel cocaine exposure therapy for relapse prevention. Biol Psychiatry 70: 593-598. 
Moghaddam B (1993). Stress preferentially increases extraneuronal levels of excitatory amino acids in the prefrontal cortex: comparison to hippocampus and basal ganglia. J Neurochem 60: $1650-1657$.

Morales-Medina JC, Sanchez F, Flores G, Dumont Y, Quirion R (2009). Morphological reorganization after repeated corticosterone administration in the hippocampus, nucleus accumbens and amygdala in the rat. J Chem Neuroanat 38: 266-272.

Moussawi K, Pacchioni A, Moran M, Olive MF, Gass JT, Lavin A et al (2009). N-Acetylcysteine reverses cocaine-induced metaplasticity. Nat Neurosci 12: 182-189.

Niswender CM, Conn PJ (2010). Metabotropic glutamate receptors: physiology, pharmacology, and disease. Annu Rev Pharmacol Toxicol 50: 295-322.

Olive MF (2009). Metabotropic glutamate receptor ligands as potential therapeutics for addiction. Curr Drug Abuse Rev 2: 8398.

Paxinos G, Watson C (1998). The Rat Brain In Stereotaxic Coordinates. 4th edn Academic Press: London.

Perrotti LI, Hadeishi Y, Ulery PG, Barrot M, Monteggia L, Duman RS et al (2004). Induction of deltaFosB in rewardrelated brain structures after chronic stress. J Neurosci 24: 10594-10602.

Phillipson OT, Griffiths AC (1985). The topographic order of inputs to nucleus accumbens in the rat. Neuroscience 16: 275-296.

Pin JP, Galvez T, Prezeau L (2003). Evolution, structure, and activation mechanism of family $3 / \mathrm{C}$ G-protein-coupled receptors. Pharmacol Ther 98: 325-354.

Popoli M, Yan Z, McEwen BS, Sanacora G (2012). The stressed synapse: the impact of stress and glucocorticoids on glutamate transmission. Nat Rev Neurosci 13: 22-37.

Rainer Q, Xia L, Guilloux JP, Gabriel C, Mocaer E, Hen R et al (2011). Beneficial behavioural and neurogenic effects of agomelatine in a model of depression/anxiety. Int J Neuropsychopharmacol 15: 1-15.

Reichel CM, Schwendt M, McGinty JF, Olive MF, See RE (2011). Loss of object recognition memory produced by extended access to methamphetamine self-administration is reversed by positive allosteric modulation of metabotropic glutamate receptor 5 . Neuropsychopharmacology 36: 782-792.

Schenk S, Partridge B (1999). Cocaine-seeking produced by experimenter-administered drug injections: dose-effect relationships in rats. Psychopharmacology 147: 285-290.

Schotanus SM, Chergui K (2008). Dopamine D1 receptors and group I metabotropic glutamate receptors contribute to the induction of long-term potentiation in the nucleus accumbens. Neuropharmacology 54: 837-844.

Sequeira-Cordero A, Mora-Gallegos A, Cuenca-Berger P, Fornaguera-Trias J (2014). Individual differences in the forced swimming test and neurochemical kinetics in the rat brain. Physiol Behav 128C: 60-69.

Shaffer C, Guo ML, Fibuch EE, Mao LM, Wang JQ (2010). Regulation of group I metabotropic glutamate receptor expression in the rat striatum and prefrontal cortex in response to amphetamine in vivo. Brain Res 1326: 184-192.

Shoji H, Mizoguchi K (2010). Acute and repeated stress differentially regulates behavioral, endocrine, neural parameters relevant to emotional and stress response in young and aged rats. Behav Brain Res 211: 169-177.

Sinclair CM, Cleva RM, Hood LE, Olive MF, Gass JT (2012). mGluR5 receptors in the basolateral amygdala and nucleus accumbens regulate cue-induced reinstatement of ethanolseeking behavior. Pharmacol Biochem Behav 101: 329-335.

Skorzewska A, Bidzinski A, Hamed A, Lehner M, Turzynska D, Sobolewska A et al (2007). Changes in hippocampal amino acid concentrations after chronic administration of corticosterone. Pharmacol Rep 59: 763-772.

Soderpalm AH, de Wit H (2002). Effects of stress and alcohol on subjective state in humans. Alcohol Clin Exp Res 26: 818-826.

Stauffer SR (2011). Progress toward positive allosteric modulators of the metabotropic glutamate receptor subtype 5 ( $\mathrm{mGlu}(5))$. ACS Chem Neurosci 2: 450-470.

Stefani MR, Moghaddam B (2010). Activation of type 5 metabotropic glutamate receptors attenuates deficits in cognitive flexibility induced by NMDA receptor blockade. Eur J Pharmacol 639: 26-32.

Stolerman I (1992). Drugs of abuse: behavioural principles, methods and terms. Trends Pharmacol Sci 13: 170-176.

Stolerman IP, Childs E, Ford MM, Grant KA (2011). Role of training dose in drug discrimination: a review. Behav Pharmacol 22: $415-429$.

Szumlinski KK, Ary AW, Lominac KD (2008). Homers regulate drug-induced neuroplasticity: implications for addiction Biochem Pharmacol 75: 112-133.

Tidey JW, Miczek KA (1997). Acquisition of cocaine selfadministration after social stress: role of accumbens dopamine. Psychopharmacology 130: 203-212.

Tomita M, Katsuyama H, Watanabe Y, Shibaike Y, Yoshinari H, Tee JW et al (2013). c-Fos immunoreactivity of neural cells in intoxication due to high-dose methamphetamine. J Toxicol Sci 38: $671-678$.

Vales K, Svoboda J, Benkovicova K, Bubenikova-Valesova V, Stuchlik A (2010). The difference in effect of mGlu2/3 and mGlu5 receptor agonists on cognitive impairment induced by MK-801. Eur J Pharmacol 639: 91-98.

Venero C, Borrell J (1999). Rapid glucocorticoid effects on excitatory amino acid levels in the hippocampus: a microdialysis study in freely moving rats. Eur J Neurosci 11: 2465-2473.

Wang W, Sun D, Pan B, Roberts CJ, Sun X, Hillard CJ et al (2010). Deficiency in endocannabinoid signaling in the nucleus accumbens induced by chronic unpredictable stress. Neuropsychopharmacology 35: 2249-2261.

Wieronska JM, Branski P, Szewczyk B, Palucha A, Papp M, Gruca P et al (2001). Changes in the expression of metabotropic glutamate receptor 5 (mGluR5) in the rat hippocampus in an animal model of depression. Polish J Pharmacol 53: 659-662.

Wise RA, Wang B, You ZB (2008). Cocaine serves as a peripheral interoceptive conditioned stimulus for central glutamate and dopamine release. PLoS One 3: e2846.

Witkin JM, Marek GJ, Johnson BG, Schoepp DD (2007). Metabotropic glutamate receptors in the control of mood disorders. CNS Neurol Disord Drug Targets 6: 87-100.

Xiao B, Tu JC, Worley PF (2000). Homer: a link between neural activity and glutamate receptor function. Curr Opin Neurobiol 10: $370-374$.

Supplementary Information accompanies the paper on the Neuropsychopharmacology website (http://www.nature.com/npp) 